\title{
Microwave and Millimeter-Wave High-Q Micromachined Resonators
}

\author{
Andrew R. Brown, ${ }^{1}$ Pierre Blondy, ${ }^{2}$ Gabriel M. Rebeiz ${ }^{1}$ \\ ${ }^{1}$ Radiation Laboratory, Department of Electrical Engineering, University of Michigan, Ann Arbor, \\ Michigan 48109; e-mail: mscout@engin.umich.edu and rebeiz@engin.umich.edu \\ 2 IRCOM, University of Limoges, Limoges, France; e-mail: pblondy@ircom.unilim.fr
}

Received 29 June 1998; revised 19 December 1998

\begin{abstract}
Alternative techniques for integrating high-quality factor resonators using micromachining techniques have been investigated. Two methods are presented which include suspending microstrip lines on thin dielectric membranes, resulting in an effective dielectric constant of near unity, and integrating three-dimensional micromachined waveguide cavity resonators with planar feedlines. These resonators show large improvements in quality factor over conventional techniques, and more importantly, allow for planar integration in complex systems. Resonators were fabricated in suspended microstrip at 29,37 , and $62 \mathrm{GHz}$ with quality factors of over 450 with very close agreement between simulated and measured results. An integrated micromachined cavity resonator was also fabricated with a $\mathrm{TE}_{011}$ resonance quality factor of 1117 at $24 \mathrm{GHz}$ and a $\mathrm{TE}_{021}$ resonance quality factor of 1163 at $38 \mathrm{GHz}$. To the authors' knowledge, these are the highest quality factor planar resonators without the use of superconductive materials, and can be used in microwave and millimeter-wave low-loss filters and low-phase-noise oscillators. (c) 1999 John Wiley \& Sons, Inc. Int J RF and Microwave CAE 9: 326-337, 1999.
\end{abstract}

Keywords: millimeter wave; high- $Q$ resonators; micromachining; packaging techniques

\section{INTRODUCTION}

Microwave and millimeter-wave communication systems are expanding rapidly as they offer many advantages over conventional wireless links. They allow the use of very wideband radio links suitable for intersatellite and personal communications. Commercially available systems are under development at $28 \mathrm{GHz}$ for the local multipoint distribution system (LMDS) [1], the PCS networks at $38 \mathrm{GHz}[2,3]$, and also a new short-range telecommunication band at $60 \mathrm{GHz}$. Commercial systems demand high yield and the ability to fabricate large volumes of systems using low-cost techniques.

Correspondence to: A. R. Brown

This work was supported by the United States Army Research Office ASSERT under Contract DAAH04-95-1-0205.
Current millimeter-wave wireless front-end transceivers use a hybrid approach with a combination of waveguide components, solid-state devices, and dielectric resonators (Fig. 1). All of the active components (LNA, power amplifier, mixer) are based on solid-state technology, and are implemented using planar MMICs. However, the diplexer and other filters are implemented either using high- $Q$ structures such as resonant waveguide cavity filters or dielectric resonator filters. These expensive components are needed in order to have a low insertion loss, high out-of-band rejection, and high channel-to-channel isolation. The theoretical insertion loss of a filter is given by [4]:

$$
\Delta L_{A}(\mathrm{~dB}) \approx 8.686 \frac{c_{n}}{\bar{\omega} Q_{u}}
$$


where $\Delta L_{A}(\mathrm{~dB})$ is the in-band insertion loss, $c_{n}$ is the filter prototype coefficient and is a function of the number of poles and passband ripple of the filter, $\bar{\omega}$ is the filter fractional bandwidth, and $Q_{u}$ is the unloaded quality factor of the resonator. Figure 2 shows the impact of the quality factor on the insertion loss of a 3 and an $8 \%$ filter. It is evident that, in order to obtain high performance, a resonator unloaded $Q$ of 500 or higher should be used for a narrowband diplexer design.

The local oscillator should also exhibit a very low-phase-noise performance which is strongly dependent on the quality factor. The oscillator phase noise, using a linear approximation, is given by:

$$
N_{p n}\left(f_{m}\right)=\frac{F k T}{2 P_{a v s}}\left[1+\left(\frac{1}{2 Q_{L}}\right)^{2}\left(\frac{f_{0}}{f_{m}}\right)^{2}\right]
$$

where $F$ is the noise figure of the active circuit with the positive feedback removed, $k$ is the Boltzmann constant, $T$ is the temperature, $P_{a v s}$ is the available signal power, $Q_{L}$ is the resonator loaded $Q, f_{0}$ is the oscillation frequency, and $f_{m}$ is the frequency offset from the carrier where the noise spectral power is measured. For frequencies near the carrier, the phase noise is a function of $1 / Q^{2}$. Millimeter-wave oscillators are typically fabricated with either a waveguide cavity such as the case of Gunn or IMPATT diodes [5] or using a dielectric resonator as in the case of HEMT or HBT devices [6-8]. These resonators exhibit a $Q$ of $1000-3000$ at $30-60 \mathrm{GHz}$, and result in excellent phase-noise performance. However, excellent performance is also achieved with a micromachined resonator with a $Q$ of 500-1000.

Micromachining has been used in the past to implement high- $Q$ on-chip mechanical resonators

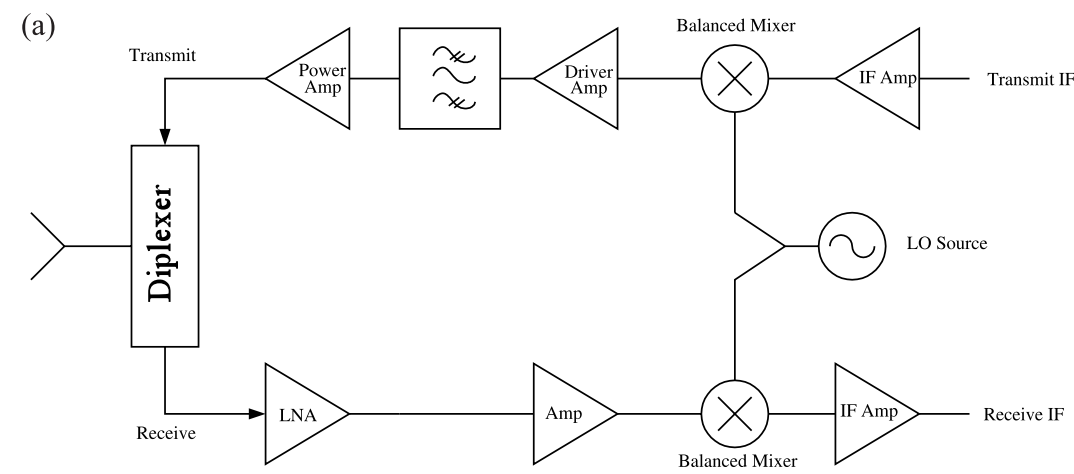

(b)

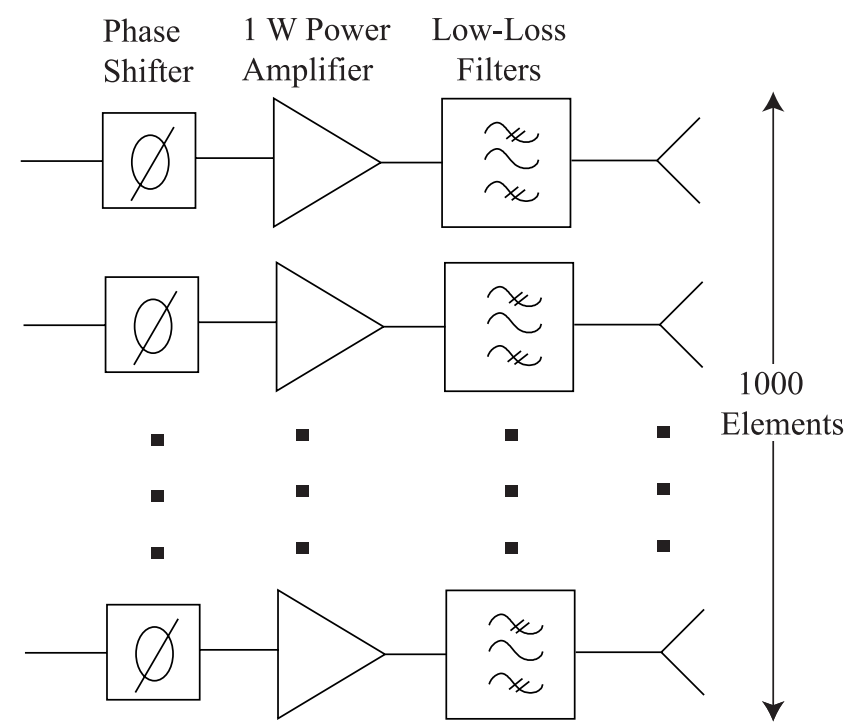

Figure 1. Typical millimeter-wave (a) transceiver front-end block diagram and (b) new-generation $K$-band phased array for satellite communications. 


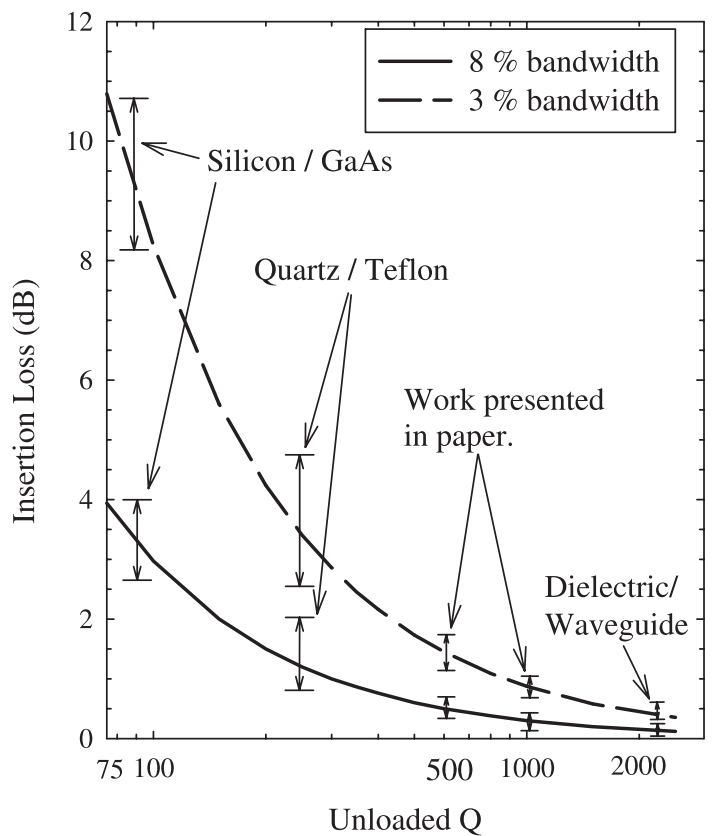

Figure 2. Theoretical insertion loss for 3 and $8 \%$ filters. The estimated type of resonator is based on data from $30-60 \mathrm{GHz}[10]$.

with an unloaded quality factor of 20,000 at 70 $\mathrm{MHz}$ on a silicon substrate [9], and it is predicted that mechanical resonators may reach $1 \mathrm{GHz}$ in the future [10]. However, the mechanical resonator approach is process intensive, requires the resonators to be vacuum sealed, and only to operate with reasonable $Q$ s at low frequencies. This paper discusses alternative methods for obtaining planar microwave and millimeter-wave high- $Q$ elements by using micromachining techniques to alter the geometry of a silicon substrate. The paper presents two methods: the first technique is based on thin dielectric (membrane) technology, and the second technique is based on threedimensional etching in a cavity formation of a silicon wafer. The micromachined resonators are fully compatible with MMIC technology, and most importantly, do not require low-loss millimeterwave transitions which are necessary in waveguide technology. Also, micromachined resonators are lithographically defined, and therefore do not require exact placement and manual tuning such as in dielectric resonator designs. It is expected that micromachining techniques will be very useful in future millimeter-wave communication and phased-array systems.

\section{DEFINITION OF QUALITY FACTOR}

The definition for the $Q$ factor is given by:

$$
Q=\omega \frac{\text { energy stored resonator }}{\text { energy dissipated }}
$$

The equivalent circuit for a distributed resonator is taken as a parallel $R L C$ lumped element model (Fig. 3). In order to excite the resonator, energy must be coupled into the resonator with either magnetic or electric coupling. To take into account the effects of input/output loading, the quality factor of a structure can be broken down into different figures of merit, namely, the loaded $Q\left(Q_{L}\right)$, the unloaded $Q\left(Q_{u}\right)$, and the external $Q$ $\left(Q_{\text {ext }}\right)$. The loaded $Q$ is the measured quality factor taking into account the loading effects of the resonator itself, and also the loading of the external circuit. Only the loaded $Q$ of a resonator can actually be measured, and the unloaded $Q$ and the external $Q$ of a resonator have to be extrapolated.

There are two main resonator configurations typically encountered in microwave and millimeter-wave circuits, namely, the bandpass and the bandstop configurations. The bandpass configuration (Fig. 4a) uses the resonator as a singleresonator bandpass filter reflecting power at frequencies away from resonance and passing power at frequencies at resonance. The bandstop configuration (Fig. 4b) allows power to pass by the resonator away from resonance, and then absorbs power from the line at resonance, increasing the effective impedance and causing more reflection.

For a resonator in a bandpass (bandstop) configuration with a resistive matched source and load impedance, the loaded quality factor can be obtained from measuring the $3 \mathrm{~dB}$ bandwidth of the $S_{21}\left(S_{11}\right)$ as shown below:

$$
Q_{L}=\frac{f_{0}}{\Delta f_{3 \mathrm{~dB}}}
$$

where $f_{0}$ is the resonant frequency and $\Delta f_{3 \mathrm{~dB}}$ is

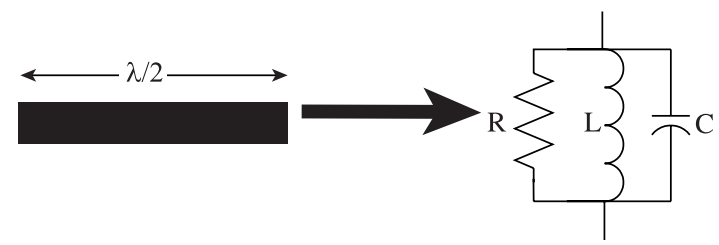

Figure 3. Equivalent parallel $R L C$ model for distributed resonator. 
(a)

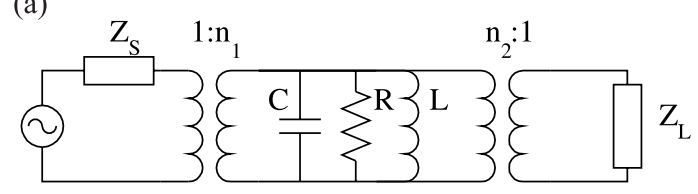

(b)

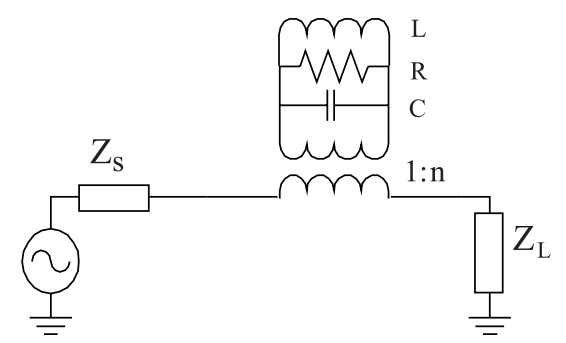

Figure 4. Typical resonator configurations for magnetic coupling in (a) bandpass and (b) bandstop configuration.

the $3 \mathrm{~dB}$ bandwidth of the $S_{21}\left(S_{11}\right)$ response. The external $Q$ can then be obtained from the loaded $Q$ and the insertion loss of the resonator at the resonant frequency by solving:

$$
Q_{\mathrm{ext}}=\frac{Q_{L}}{S_{21}\left(f_{0}\right)} \quad \text { or } \quad\left(Q_{\mathrm{ext}}=\frac{Q_{L}}{S_{11}\left(f_{0}\right)}\right)
$$

where $S_{21}\left(f_{0}\right)$ [or $S_{11}\left(f_{0}\right)$ ] is the transmission (reflection) coefficient at resonance for the bandpass (bandstop) resonator and is measured in linear scale. The unloaded $Q$ can be found from:

$$
Q_{u}=\frac{Q_{L}}{1-S_{21}\left(f_{0}\right)} \quad \text { or } \quad\left(Q_{u}=\frac{Q_{L}}{1-S_{11}\left(f_{0}\right)}\right)
$$

For accurate measurements, the coupling should be weak so that any influence of the loss of the feedline structure does not have a strong impact on the unloaded $Q$ extrapolation.

The three definitions for the quality factor for both the bandpass and the bandstop configurations are given by:

$$
\frac{1}{Q_{u}}=\frac{1}{Q_{L}}-\frac{1}{Q_{\mathrm{ext}}}
$$

\section{MEMBRANE-SUPPORTED SUSPENDED MICROSTRIP RESONATORS}

For the case of a distributed transmission line resonator, such as a microstrip or CPW res- onator, the quality factor can be found by:

$$
Q=\frac{\pi}{\lambda \alpha}
$$

where $\lambda$ is the guided wavelength and $\alpha$ is the attenuation in nepers per meter, including attenuation by radiation, substrate loss, and ohmic loss. Distributed resonators using microstrip structures on conventional substrates have been used extensively for applications at $X$-band and below with reasonable values of $Q$ (100-150 on quartz/Teflon). However, with increasing frequency, thinner substrates must be used to reduce radiation loss in substrate modes. This results in narrow line dimensions for a given impedance, which greatly increases the ohmic loss, and drastically reduces the resonator $Q$. Micromachining techniques are used to produce a micropackaged, air dielectric line with wide transverse dimensions resulting in high- $Q$ resonators at millimeter-wave frequencies.

Membrane-supported microstrip structures are formed by removing the silicon substrate and suspending a microstrip line on a thin $(1.4 \mu \mathrm{m})$ dielectric membrane. A ground plane is formed by another micromachined substrate, and is attached to the top of the circuit. The bottom is also shielded with a third substrate (Fig. 5). For this structure, dielectric loss is eliminated with the air dielectric, the radiation loss is minimized by shielding the structure on all sides using thick via grooves to limit substrate modes, and ohmic loss is greatly reduced by allowing for very wide transverse microstrip geometries. Silicon has been used for its low-cost anisotropic etching properties. Other materials can also be used such as InP and GaAs. In the past, micromachining techniques have been successfully applied to $K$ - and $W$-band membrane-supported microstrip lines and filters $[11,12]$.

\section{A. Fabrication}

The micromachined suspended microstrip transmission line is based on a three-wafer process (Fig. 5). The top wafer will be referred to as the ground plane wafer, the middle wafer as the circuit wafer, and the bottom wafer as the shielding wafer. For the circuit wafer, a stress-compensated $1.4 \mu \mathrm{m}$ membrane layer consisting of $\mathrm{SiO}_{2} / \mathrm{Si}_{3} \mathrm{~N}_{4} / \mathrm{SiO}_{2}(7000 \AA / 4000 \AA / 3000 \AA)$ is deposited on a high-resistivity $525 \mu \mathrm{m}$ thick silicon substrate using thermal oxidation and low- 
Upper Cavity Wafer

Microstrip Ground Plane

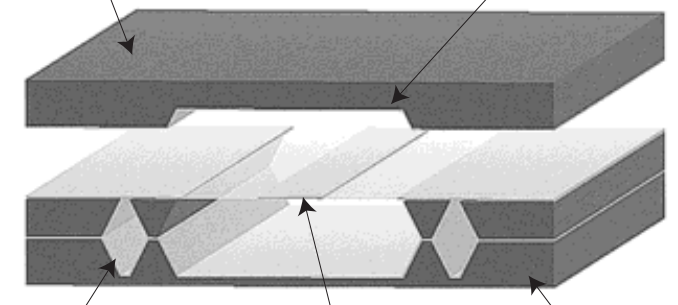

Via Grooves

Microstrip on

Membrane

Lower Cavity Wafer

Figure 5. Transverse section of the microstrip structure.

pressure chemical vapor deposition. This process deposits the thin film on both sides of the silicon wafer, allowing for a membrane on the top side of the wafer and a good etch mask for the silicon removal on the back side. The thicknesses of the $\mathrm{SiO}_{2} / \mathrm{Si}_{3} \mathrm{~N}_{4}$ layers are optimized by using a deflection method [13] to balance the net stress, leaving the membrane in slight tension (5-10 $\mathrm{MPa}$ ) to result in flat and rigid membranes. After the membrane is deposited, the circuit is patterned on the top side of the wafer using either a standard $2 \mu \mathrm{m}$ gold electroplating technique or a $1 \mu \mathrm{m}$ evaporated gold and lift-off procedure, depending on the operating frequency of the resonator. Other circuit components such as thin-film capacitors, resistors, or air bridges can also be included at this time. Next, an opening is defined on the back side of the wafer under the resonators and areas where via holes are to be formed by etching the back-side membrane with an RIE machine. The silicon is then completely etched under the circuit to the dielectric membrane which acts as an excellent etch stop. The etchant used in this work was a solution of $12.5 \%$ tetramethyl ammonium hydroxide (TMAH) and water [14]. This solution has a $1.1 \mu \mathrm{m} / \mathrm{min}$ etch rate for the $\langle 100\rangle$ crystal plane with a $\langle 100\rangle:\langle 111\rangle$ selectivity of 25:1.

The bottom shielding wafer and the top ground plane wafer are $525 \mu \mathrm{m}$ thick low-resistivity wafers with a thermal oxide that acts as an etch mask. The bottom wafer is etched down $400 \mu \mathrm{m}$, and metalized with gold to prevent radiation. The top ground plane wafer is formed by double-side etching. First, a selective etch is performed on the upper side of the top wafer to begin probe window openings. Next, the lower side is patterned, and the wafer is etched on both sides to open the probe windows and to form the upper cavity ground plane. The three wafers are finally assem- bled using conductive silver epoxy [15] to form the complete resonator.

\section{B. Simulation and Measurement}

Several resonators were constructed using both the bandpass and bandstop configurations. In all cases, the resonators are $\lambda_{0} / 2$ microstrip lines that are weakly coupled by capacitive coupling gaps or magnetically coupled by parallel coupled lines.

A single resonator was fabricated at $29 \mathrm{GHz}$ in a bandstop configuration (Fig. 6). The ground plane height is $250 \mu \mathrm{m}$ with an $800 \mu \mathrm{m}$ wide conductor. The metal thickness of the conductor is $2 \mu \mathrm{m}$ of electroplated gold (four skin depths at $29 \mathrm{GHz}$ ). The shielding cavity is $800 \mu \mathrm{m}$ away from the resonator. In order to conserve membrane space, the resonator was bent in a U-shape using optimal miters. The length of the resonator was adjusted for the correct resonant frequency to $29.0 \mathrm{GHz}$ by using a $2 \frac{1}{2} \mathrm{D}$ moment-method package IE3D [16]. The measured resonance was at $28.7 \mathrm{GHz}$, showing a $1 \%$ shift in the resonant frequency. This is due to the fringing capacitance to the sidewalls of the cavity that was not modeled with the full-wave analysis technique.

The suspended microstrip feedline is first measured without the resonator to study the effect of the CPW-to-microstrip transition. The microstrip line is $1025 \mu \mathrm{m}$ wide, resulting in an impedance of $50 \Omega$ over a $250 \mu \mathrm{m}$ ground plane. The transition can be modeled with a series inductor to account for the extra inductance between the $\mathrm{CPW}$ and microstrip ground planes. Figure 7 shows the measured and modeled $S_{11}$, and excellent agreement is achieved with a $0.05 \mathrm{nH}$ series inductance. Figure 7 also shows the $S_{11}$ response with the resonator present. As shown, the microstripline length is chosen to result in a good match at $27-32 \mathrm{GHz}$ with the effects of the transition discontinuity. The measured loaded $Q$ was 190 with a coupling $-4.6 \mathrm{~dB}$, giving an extracted unloaded $Q$ of 460 at $28.7 \mathrm{GHz}$.

Three different resonators were fabricated in bandpass configurations at 37 and $60 \mathrm{GHz}$ (Fig. 8). At $37 \mathrm{GHz}$, the resonator has a ground plane height of $200 \mu \mathrm{m}$ and a line width and thickness of 700 and $2 \mu \mathrm{m}$, respectively. The extracted unloaded $Q$ based on measurements is 412 at 37 GHz. Two different resonators were also fabricated at $60 \mathrm{GHz}$. Both resonators have a ground plane height of $250 \mu \mathrm{m}$ with widths of 500 and $700 \mu \mathrm{m}$. The resonator thickness is $1 \mu \mathrm{m}$ of 


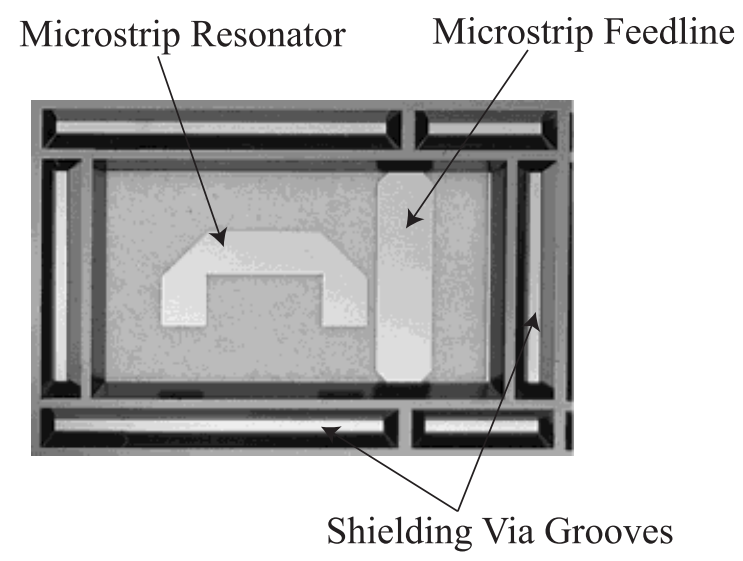

(a)

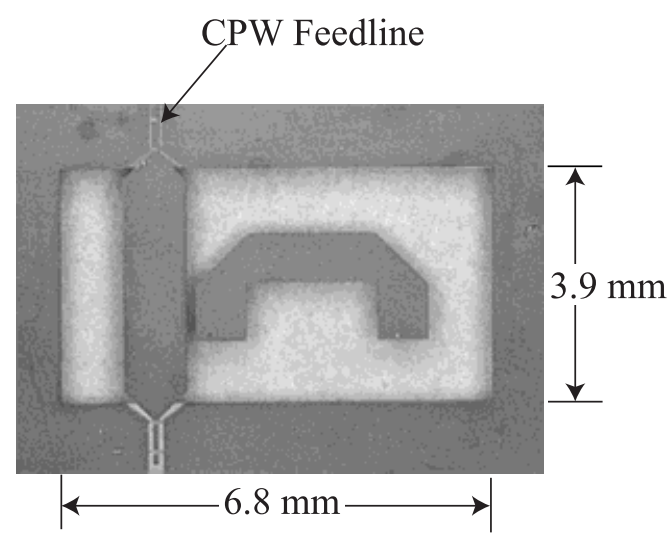

(b)

Figure 6. Circuit wafer of a $29 \mathrm{GHz}$ microstrip resonator in bandstop configuration. (a) Bottom view. (b) Top view.

evaporated gold (three skin depths at $60 \mathrm{GHz}$ ). The $500 \mu \mathrm{m}$ lines had an extracted unloaded $Q$ of 454 , and the $700 \mu \mathrm{m}$ lines had an extracted unloaded $Q$ of 503. The repeatability of the measurements is to within $1 \%$ in $Q$ and to within $0.03 \%$ in frequency. Some of these resonators have been used in state-of-the-art low-loss filters at 37 and $60 \mathrm{GHz}$ [17].

The four structures described above were simulated using method-of-moments-based HPMomentum [18], a finite-element tool [19], an empirical model from Linecalc [20], and a code based on the surface impedance method [21] called Simian [22]. For the simulations, the conductivity of the gold lines was assumed to be $3.9 \times 10^{7} \Omega^{-1} \cdot \mathrm{m}^{-1}$. The results for the simulations and measurements are summarized in Table I. The values based on Linecalc, MoM, and FEM all overestimate the value for the unloaded $Q$ considerably. However, Simian models the loss very accurately, and is within $5 \%$ of the measured values. The measured $Q_{u}$ values are $10 \times$ larger than microstrip resonators on GaAs or silicon, and $2 \times$ larger than corresponding resonators on low-dielectric-constant substrates such as Teflon [10].

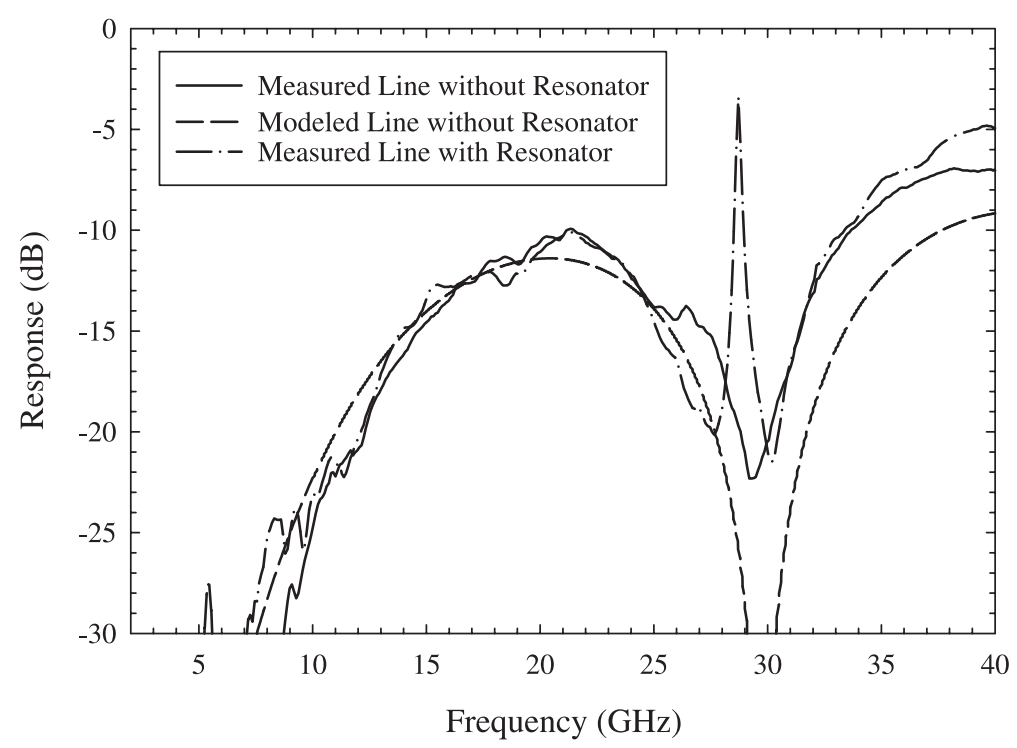

Figure 7. Measured $S_{11}$ of bandstop resonator including effects of transition. 


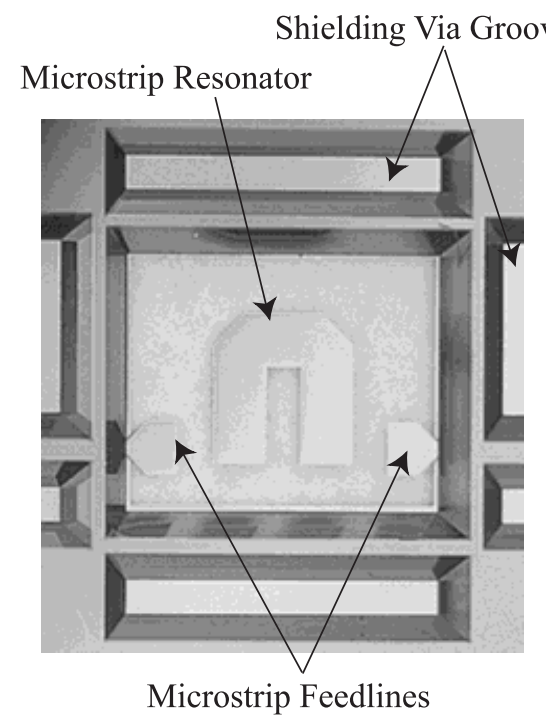

(a)

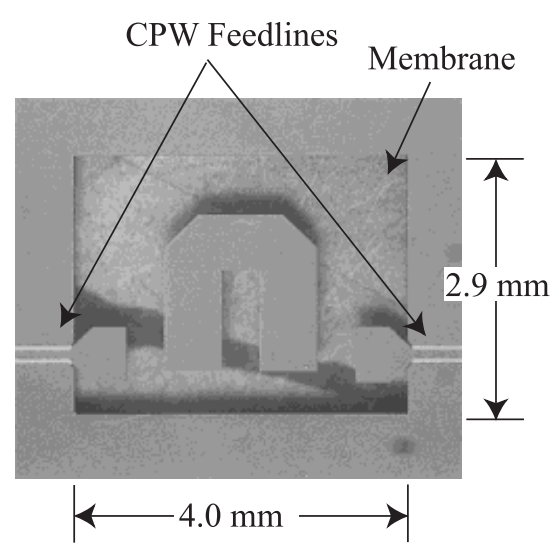

(b)

Figure 8. Bandpass configuration of $37 \mathrm{GHz}$ micromachined microstrip resonator. (a) Bottom view. (b) Top view.

Design curves for the $Q$-factor were generated using Simian (Fig. 9) for both the $30 \mathrm{GHz}(t=2$ $\mu \mathrm{m}$ gold $)$ and $60 \mathrm{GHz}(t=2 \mu \mathrm{m}$ gold $)$ resonators. For the $60 \mathrm{GHz}$ calculations, increasing the metal thickness to $2 \mu \mathrm{m}$ will result in a $2-3 \%$ improvement in $Q$. The value of the resonator $Q$ is very sensitive to the ground plane height. Since the cavity sidewalls are far from the line, the structure is insensitive to $X-Y$ alignment. However, with increasing height $(h>250 \mu \mathrm{m})$, the CPW-to-membrane transition becomes more difficult with added transition inductance from the CPW ground to the microstrip ground plane. Also, increasing the width of the lines past 800-900 $\mu \mathrm{m}$ results in very wide transmission lines, and makes circuit modeling and design more difficult. (See Fig. 10.) Therefore, practical considerations will limit the $Q$ of such resonators to 450-550 for most applications.

\section{INTEGRATED MICROMACHINED CAVITY RESONATORS}

The quality factor of the suspended microstripline shows a large improvement over conventional microstriplines, but in many cases, an unloaded quality factor of 500 is still not enough. One way to increase the resonator $Q$ is to integrate miniature three-dimensional cavities in the silicon substrate.

The integrated micromachined waveguide resonator was first demonstrated at $10 \mathrm{GHz}$ for the case of very strong transmission coupling using a two-wafer process with a slot-aperture coupling [23]. The slot was excited by a microstripline above it. This method requires two wafers: one for the microstrip-to-slot transition, and the second for the actual cavity (Fig. 11a). However, the $Q$ of the resonator is strongly related to the

TABLE I. Comparison Between Measured and Simulated Values of Unloaded $Q$

\begin{tabular}{lcccc}
\hline & $w=800 \mu \mathrm{m}$ & $w=700 \mu \mathrm{m}$ & $w=700 \mu \mathrm{m}$ & $w=500 \mu \mathrm{m}$ \\
& $h=250 \mu \mathrm{m}$ & $h=200 \mu \mathrm{m}$ & $h=250 \mu \mathrm{m}$ & $\begin{array}{c}w=250 \mu \mathrm{m} \\
f_{0}=62 \mathrm{GHz}\end{array}$ \\
& $f_{0}=29 \mathrm{GHz}$ & $f_{0}=37 \mathrm{GHz}$ & $f_{0}=62 \mathrm{GHz}$ & $f_{0}=$ \\
\hline Linecalc [20] & 528 & 474 & 665 & 606 \\
FEM [19] & & 442 & 661 & 610 \\
MoM [18] & 550 & 470 & 613 & 536 \\
Simian [22] & 450 & 403 & 525 & 474 \\
Measured & 460 & 412 & 503 & 454 \\
$\alpha(\mathrm{dB} / \mathrm{cm})$ & 0.057 & 0.082 & 0.112 & 0.119 \\
\hline
\end{tabular}




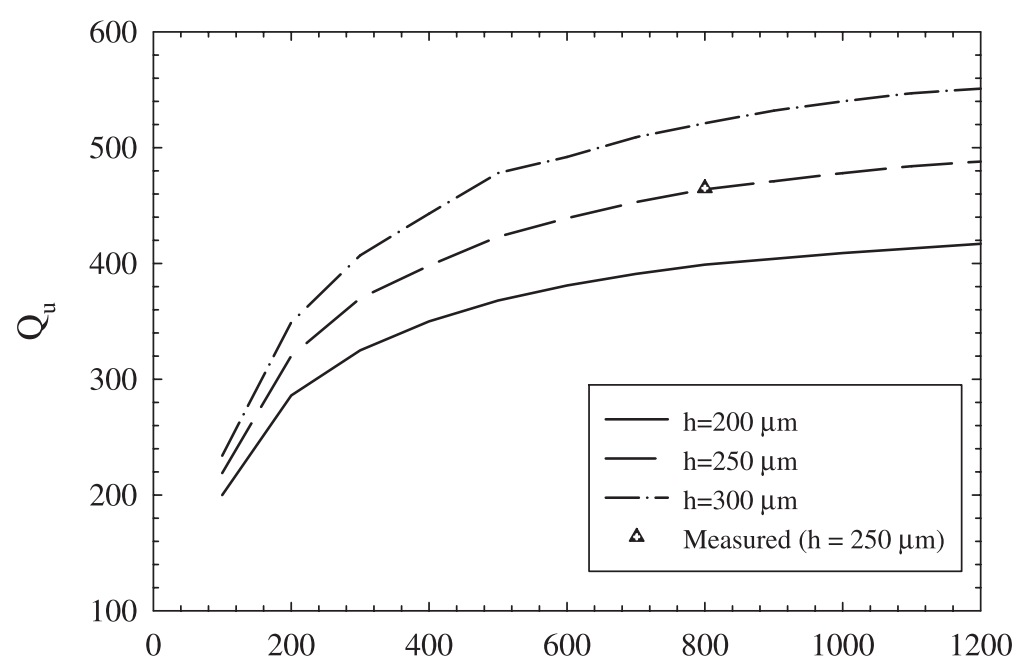

Conductor Width $(\mu \mathrm{m})$

(a)

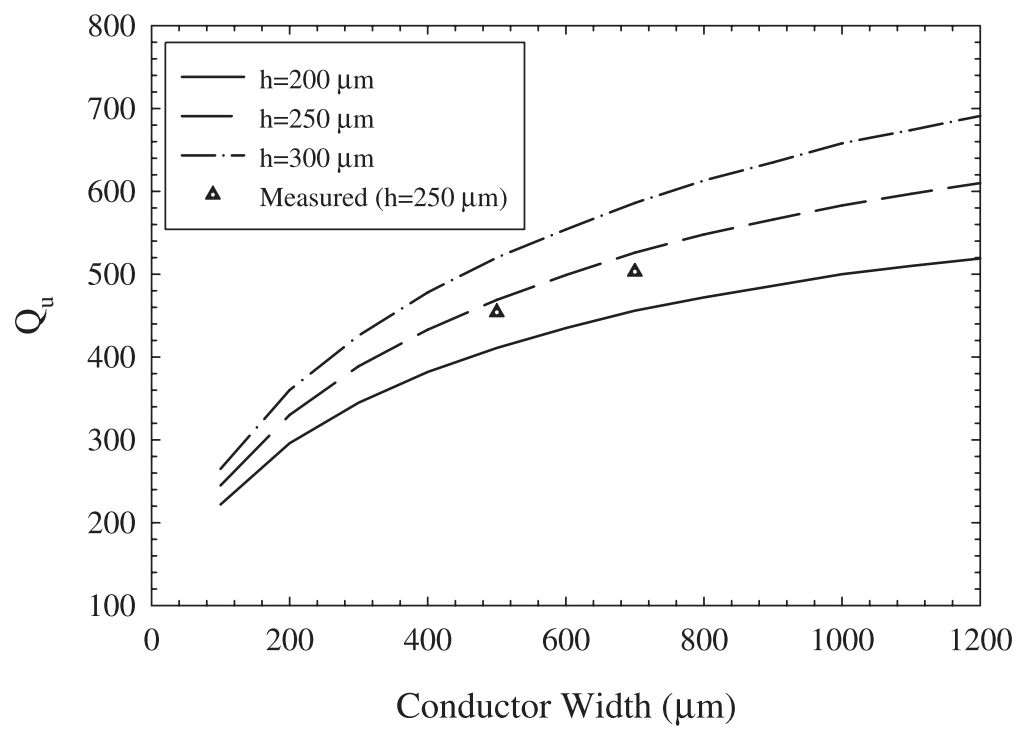

(b)

Figure 9. Simulated (Simian) microstrip resonator $Q$ as a function of height and strip width at (a) $30 \mathrm{GHz}$ with $2 \mu \mathrm{m}$ thick lines and (b) $60 \mathrm{GHz} 1 \mu \mathrm{m}$ thick lines.

thickness of the cavity which, in this case, is limited to a standard thickness wafer. Also, at millimeter-wave frequencies, the microstripline must be integrated on a thin substrate $(250 \mu \mathrm{m}$ at $30 \mathrm{GHz}, 150 \mu \mathrm{m}$ at $60 \mathrm{GHz}$, etc.) with the expected additional circuit losses.

A new approach is presented for feeding the cavity that increases the quality factor and simplifies the fabrication. The cavity is constructed from two standard thickness $(525 \mu \mathrm{m})$ silicon wafers etched $450 \mu \mathrm{m}$ down. A CPW line is defined on the bottom wafer to the edge of the cavity, and a wire bond is placed from the end of the CPW line to the base of the cavity. This acts as an electric loop triggering the dominant $\mathrm{TE}_{011}$-mode at resonance. The amount of coupling is controlled by the length of the wire bond and the number of bonds placed. The top cavity is identical to the bottom cavity, except that a groove (called a "mouse hole") is etched above the CPW line on the bottom cavity. The groove is to prevent shorting the CPW line when the two wafers are bonded together (Fig. 11b). The two wafers are bonded together with conductive epoxy. For minimal cavity disturbance, the feedlines and wire bonds are placed where the current on the sidewalls of the 


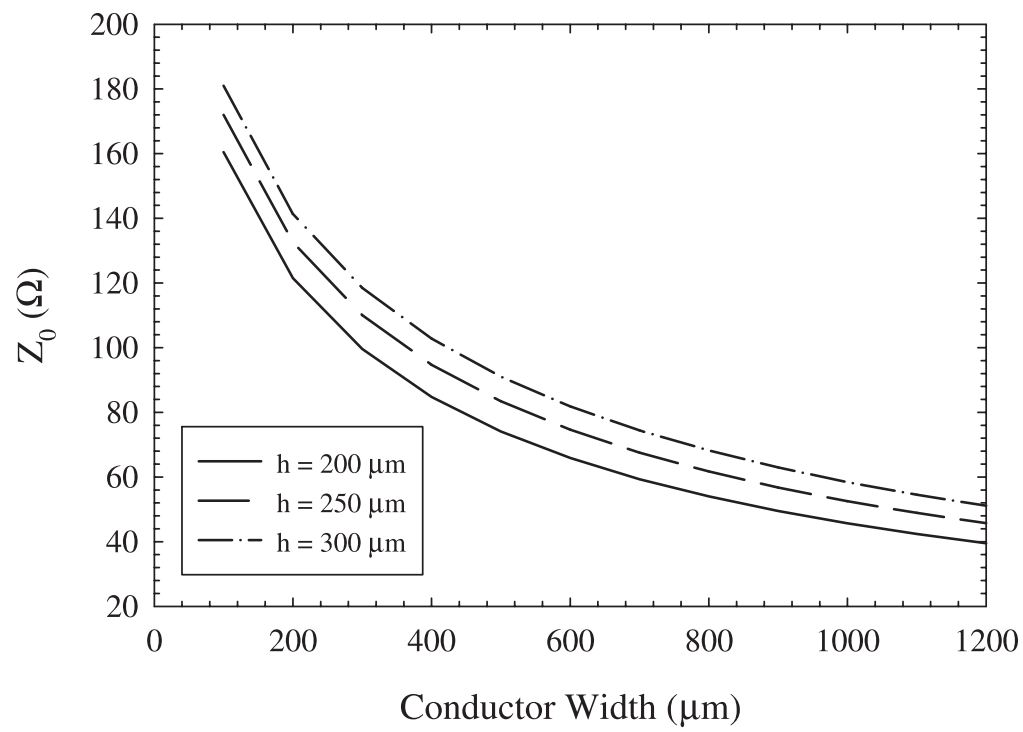

Figure 10. Simulated impedance value for suspended microstrip resonator as a function of height and strip width.

cavity is at a minimum. For the case of a rectangular cavity, the corners have a current null. Using this method, the maximum operating frequency is set by the dimensions of the CPW feedlines, and not the substrate thickness, and the $Q$ is increased by increasing the cavity height by a factor of 2 using standard thickness substrates.

\section{A. Fabrication}

A thermal oxide is grown on two standard-thickness silicon wafers to act as an etch mask for the micromachining process. The bottom wafer that contains the feedlines is a high-resistivity substrate $(2000 \Omega \cdot \mathrm{cm})$, and the top wafer is a low-

(a)

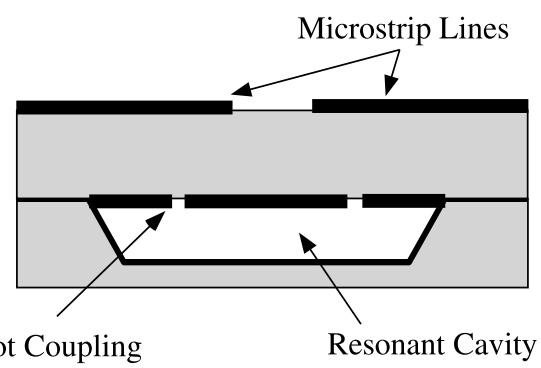

(b)

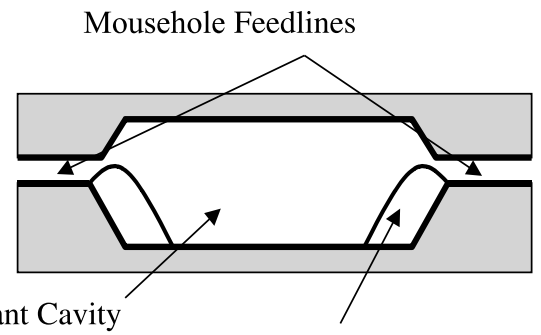

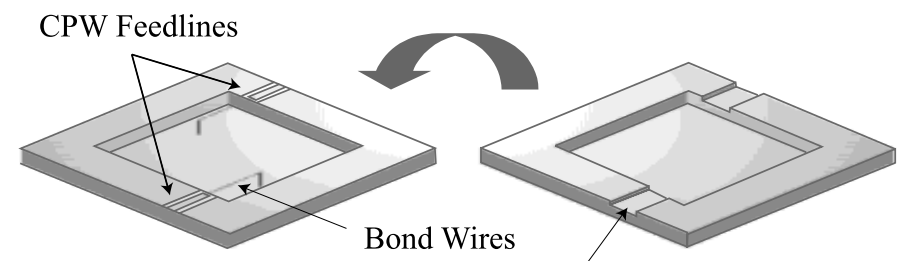

Mousehole Structure

Bond Wires (Triggers $\mathrm{TE}_{011}$ mode)

Figure 11. Side view of micromachined cavity resonator. (a) Method used by Papapolymerou et al. (b) Method used in this work. 


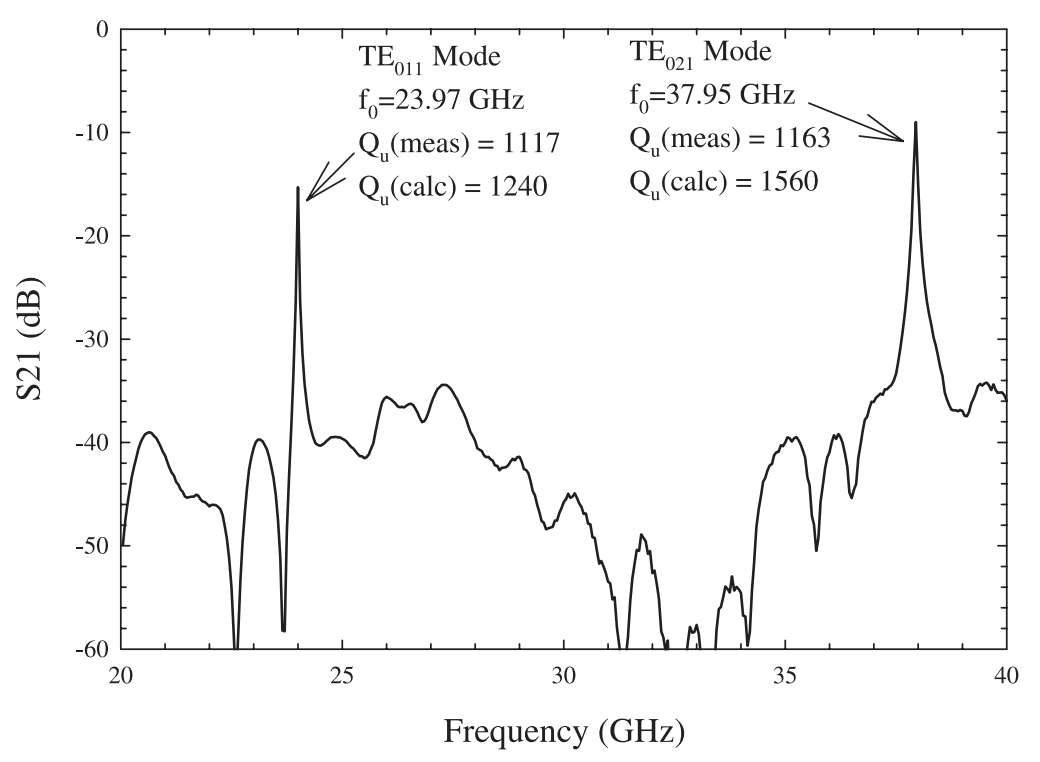

Figure 12. Two-wafer micromachined cavity resonator.

resistivity substrate $(5-7 \Omega \cdot \mathrm{cm})$. The CPW feedlines are deposited on the bottom wafer using a gold electroplating process. Next, the oxide where the cavity is to be etched is removed with a wet chemical etch. The cavity resonator is defined 500 $\mu \mathrm{m}$ away from the end of the CPW feedline. The designed cavity dimensions are $8.84 \mathrm{~mm} \times 8.84$ $\mathrm{mm}$ for a $\mathrm{TE}_{011}$ resonant frequency of $24 \mathrm{GHz}$. The cavity is then etched $450 \mu \mathrm{m}$ in an anisotropic silicon etchant (TMAH). The etchant has a selectivity to the $\langle 100\rangle:\langle 111\rangle$ crystal plane of approximately 25:1. This gives an undercut of roughly 15 $\mu \mathrm{m}$, leaving a small $\mathrm{SiO}_{2}$ lip. It is essential to remove this lip in BHF to eliminate any gold shadowing due to the lip, and thus result in a very low $Q$. The CPW feedlines are then masked with photoresist, and the entire cavity is metalized with a $3 \mu \mathrm{m}$ layer of sputtered and electroplated gold. Four $18 \mu \mathrm{m}$ thick gold wire bonds in parallel are used to excite the cavity. The wire bonds were approximately $1.2 \mathrm{~mm}$ long, placed from the feedline in the corner of the cavity diagonally toward the center of the cavity with a calculated series inductance of $0.5 \mathrm{nH}$.

The top wafer is fabricated using a two-step etch to allow for the "mouse holes" above the CPW feedlines. First, the cavity and the feedline mouse holes are patterned, and the oxide is etched halfway through. Next, the cavity alone is patterned, and the remaining oxide covering the cavity is etched, exposing the bare silicon. The substrate is then placed in the anisotropic etchant, and the cavity is etched $350 \mu \mathrm{m}$ down. The re- maining oxide covering the feedline mouseholes is carefully etched away, making sure not to completely etch the oxide protecting the surrounding areas. Again, the substrate is placed in the silicon etchant for another $100 \mu \mathrm{m}$ of etching, yielding a cavity depth of $450 \mu \mathrm{m}$ and a mousehole depth of $100 \mu \mathrm{m}$. The thermal oxide is completely stripped off, eliminating the lip formed from the undercut of the silicon etching. The top wafer is then metalized with a $3 \mu \mathrm{m}$ layer of sputtered and electroplated gold. The two substrates are bonded using conductive epoxy.

\section{B. Simulation}

The unloaded quality factor of a resonant cavity is found from [24]:

$$
Q_{u}=\omega \frac{W_{m}+W_{e}}{P_{l}}
$$

where $W_{m}$ and $W_{e}$ are the energies stored in the magnetic and electric fields, respectively, and $P_{l}$ is the net power dissipated. In the case of the air-filled cavity, the net power dissipated is purely the ohmic loss from the currents on the sides of the resonator. By neglecting the effects of the nonvertical sidewalls, the effects of the mousehole, any imperfections in bonding, and by assuming that all of the current is concentrated in one skin depth, the quality factors for different resonances can be easily calculated using the known current and field distribution for a given cavity 
mode. The unloaded quality factor for the first two modes is 1240 for the $\mathrm{TE}_{011}$ at $24 \mathrm{GHz}$ and 1560 for the $\mathrm{TE}_{021}$ mode at $38 \mathrm{GHz}$ using the conductivity of gold as $3.9 \times 10^{7} \Omega^{-1} \cdot \mathrm{m}^{-1}$.

\section{Measurements}

The cavity resonator was measured with an HP8510C using an SOLT calibration with the probe tips as the calibrated reference plane. The measured $\mathrm{TE}_{011}$ resonant mode was $23.97 \mathrm{GHz}$, which is in excellent agreement with the designed value of $24 \mathrm{GHz}$. At resonance, the measured $S_{21}$ is $-15.2 \mathrm{~dB}$ with a loaded quality factor $\left(Q_{l}\right)$ of 909 , resulting in a $Q_{u}$ of 1100 , which is in good agreement with the calculated value of 1237 . The calculated value does not include the increase resistance from the silver epoxy bonding, the effects of the mouse holes altering the current distribution on the resonator, or the loss in the 2 $\mathrm{mm}$ CPW feedlines. The combined loss of both feedlines is $0.6 \mathrm{~dB}$. Subtracting this from the measured $S_{21}$ gives a $Q_{u}$ of 1117 . The $\mathrm{TE}_{021}$ occurs at $37.95 \mathrm{GHz}$ with a measured value of $Q_{l}$ of 690 with an $S_{21}$ of $-7.9 \mathrm{~dB}$. When the loss of the CPW feedlines $(0.75 \mathrm{~dB}$ at $38 \mathrm{GHz})$ is removed, $Q_{u}$ is 1163 . To the authors' knowledge, this is the highest $Q$ integrated resonator to date at $K$-band, and could be used in low-phase-noise millimeter-wave oscillator designs.

\section{CONCLUSIONS}

Micromachining techniques have been applied to fabricate high- $Q$ integrated resonators. The fabrication is compatible with most CMOS and MMIC processes. Micromachined suspended microstriplines exhibit quality factors in the range of $450-500$ at 29, 37, and $62 \mathrm{GHz}$ in both bandpass and bandstop configurations. Furthermore, calculations using Simian show that practical considerations limit the $Q$ of suspended microstrip resonators to 550. These resonators can be easily used in complex filter designs [17]. Integrated miniature waveguide cavity resonators have also been fabricated at $24 \mathrm{GHz}$ (dominant mode) with a quality factor of 1100-1200. The micromachined waveguide cavities can be readily scaled to $60 \mathrm{GHz}(3.5 \mathrm{~mm} \times 3.5 \mathrm{~mm} \times 800 \mu \mathrm{m}$ with a calculated $\left.Q_{u}=1600\right)$ and to $77 \mathrm{GHz}(2.8 \mathrm{~mm} \times$ $2.8 \mathrm{~mm} \times 800 \mu \mathrm{m}$ with a calculated $Q_{u}=1670$ ) using standard thickness wafers for communication systems and automotive radars.

\section{ACKNOWLEDGMENT}

The authors would like to thank Dr. G. Ponchak, NASA Lewis Research Center, for providing the 60 $\mathrm{GHz}$ measurement setup.

\section{REFERENCES}

1. D.A. Gray, A broadband wireless access system at 28 GHz, 1997 Wireless Commun Conf Proc, 1997, pp. 1-7.

2. H.H. Meinel, Commercial applications of millimeter-waves. History, present status and future trends, IEEE Trans Microwave Theory Tech 43 (1995), 1639-1653.

3. J. Burns, The application of millimetre-wave technology for personal communication networks in the United Kingdom and Europe: A technical and regulatory overview, IEEE MTT-S Int Microwave Symp Dig, 1994, pp. 635-638.

4. G.L. Matthaei, L. Young, and E.M.T. Jones, Microwave filters, impedance-matching networks, and coupling structures, Artech House, Norwood, MA, 1980.

5. S. Nagano and S. Ohnaka, A low-noise $80 \mathrm{GHz}$ silicon IMPATT oscillator highly stabilized with a transmission cavity, IEEE Trans Microwave Theory Tech MTT-22 (1974), 1152-1159.

6. M. Funabashi, T. Inoue, K. Ohata, K. Maruhashi, K. Hosoya, M. Kuzuhara, K. Kanekawa, and Y. Kobayashi, A $60 \mathrm{GHz}$ MMIC stabilized frequency source composed of a $30 \mathrm{GHz}$ DRO and a doubler, IEEE MTT-S Int Microwave Symp Dig, 1995, pp. 71-74.

7. S. Chen, S. Tadayon, T. Ho, K. Pande, P. Rice, J. Adair, and M. Ghahremani, U-band MMIC HBT DRO, IEEE Microwave Guided Wave Lett 4 (1994), 50-52.

8. H. Wang, K. Chang, L. Tran, J. Cowles, T. Block, E. Lin, G. Dow, A. Oki, D.C. Streit, and B. Allen, Low phase noise millimeter wave frequency sources using InP-based HBT MMIC technology, IEEE J Solid-State Circuits 31 (1996), 1419-1425.

9. A.N. Cleland and M.L. Roukes, Fabrication of high frequency nanometer scale mechanical resonators from bulk Si crystals, Appl Phys Lett (Oct. 1996), 2653-2655.

10. C.T.-C. Nguyen, L.P.B. Katehi, and G.M. Rebeiz, Micromachined devices for wireless communications, Proc IEEE (1998), 1756-1768.

11. G.M. Rebeiz, L.P.B. Katehi, T.M. Weller, C.-Y. Chi, and S.V. Robertson, Micromachined membrane filters for microwave and millimeter-wave applications, Int $\mathrm{J}$ Microwave Millimeter-Wave CAE (1997), 149-166. 
12. C.-Y. Chi and G.M. Rebeiz, Conductor-loss limited stripline resonator and filters, IEEE Trans Microwave Theory Tech 44 (1996), 626-630.

13. L.I. Maissel and R. Glang, Handbook of thin film technology, McGraw-Hill, New York, 1983.

14. O. Tabata, R. Asahi, H. Funabashi, K. Shimaoka, and S. Sugiyama, Anisotropic etching of silicon in TMAH solutions, Sens Actuators A (1992), 51-57.

15. Epotek H20E, Epoxy Technology, 14 Fortune Drive, Billerica, MA.

16. Zeland IE3D, Release 4.12, 1997.

17. P. Blondy, A.R. Brown, D. Cros, and G.M. Rebeiz, Low loss micromachined filters for millimeter-wave telecommunication systems, IEEE MTT-S Int Microwave Symp Dig 1998, pp. 1181-1184.

18. Hewlett Packard Momentum, Release 2.0, 1997.

19. M. Aubourg and P. Guillon, A mixed finite element formulation for microwave devices problems. Ap- plication to MIS structure, J Electromagn Waves Appl 5 (1991), 371-386.

20. Hewlett Packard Linecalc, 1997.

21. E. Tuncer, B.-T. Lee, and D.P. Neikirk, Interconnect series impedance determination using a surface ribbon method, 3rd Topical Meeting Elect Performance of Electron Packaging, Nov. 1994, pp. 250-252.

22. S. Kim, E. Tuncer, B.-T. Lee, and D.P. Neikirk, Surface impedance method for interconnect analysis, http://weewave.mer.utexas.edu/MedHome. html, 1997.

23. J. Papapolymerou, J.-C. Cheng, J. East, and L.P.B. Katehi, A micromachined high-Q X-band resonator, IEEE Microwave Guided Wave Lett 7 (1997), 168-170.

24. R.E. Collin, Foundations for microwave engineering, McGraw-Hill, New York, 1992.

\section{BIOGRAPHIES}

Andrew R. Brown received the B.S.E.E. and M.S.E.E. degrees from the University of Michigan, Ann Arbor, in 1995 and 1996, respectively. He is currently pursuing a $\mathrm{Ph} . \mathrm{D}$. in electrical engineering at the same university. His present research involves applying micromachining techniques to achieve very low-loss transmission lines and high- $Q$ resonant structures for millimeter-wave communication systems. (Photo not available.)

Pierre Blondy received the D.E.A. and Ph.D. degrees from the University of Limoges in 1995 and 1998, respectively. During the summer 1997, he was a visiting student at the University of Michigan, Ann Arbor, where he studied membrane-supported micromachined resonators and filters. He is currently with the Centre Nationale de la Recherche Scientifique (CNRS) at the IRCOM institute, University of Limoges. His research interests include electromagnetic modeling and micromachining techniques applied to millimeter-wave components. (Photo not available.)

Gabriel M. Rebeiz received the Ph.D. degree in electrical engineering from the California Institute of Technology in June 1988. He joined the faculty of the University of Michigan in September 1988, and was promoted to Full Professor in May 1998. Prof. Rebeiz received the National Science Foun- dation Presidential Young Investigator Award in April 1991 and the URSI International Isaac Koga Gold Medal Award for Outstanding International Research in August 1993. He also received the Research Excellence Award in April 1995 from the University of Michigan. Together with his students, he was the winner of Best Student Paper Awards from IEEE MTT $(98,94,92)$, and IEEE AP $(95,92)$, and received the JINA ' 90 Best Paper Award. He has held short visiting professorships at Chalmers University of Technology, Gothenburg, Sweden, Ecole Normale Superieur, Paris, France, and Tohoku University, Sendai, Japan. Prof. Rebeiz received the EECS Department Teaching Award in October 1997, was selected by the students as the 1997-1998 Eta Kappa Nu EECS Professor of the Year. He also received the College of Engineering Excellence in Teaching Award in October 1998. In June 1998, he received the Amoco Foundation Teaching Award, given yearly to one (or two) faculty at the University of Michigan, for excellence in undergraduate teaching. Prof. Rebeiz's research interests are in applying micromachining techniques and MEMS for the development of novel components and subsystems for wireless communication systems. $\mathrm{He}$ is also interested in $\mathrm{Si} / \mathrm{GaAs} \mathrm{RFIC}$ design for receiver applications, and in the development of planar antennas and microwave/ millimeter-wave front ends electronics for applications in mm-wave communication systems, automotive collisionavoidance sensors, monopulse tracking systems and phased arrays. (Photo not available.) 